\title{
FORMULAS FOR AVERAGE TRANSITION TIMES BETWEEN STATES OF THE MARKOV BIRTH-DEATH PROCESS
}

\author{
Yuriy Zhernovyi ${ }^{1}$, Bohdan Kopytko ${ }^{2}$ \\ ${ }^{I}$ Ivan Franko National University of Lviv, Lviv, Ukraine \\ ${ }^{2}$ Department of Mathematics, Czestochowa University of Technology \\ Częstochowa, Poland \\ yuriy.zhernovyy@lnu.edu.ua,bohdan.kopytko@im.pcz.pl
}

Received: 6 October 2021; Accepted: 25 November 2021

\begin{abstract}
In this paper, we consider Markov birth-death processes with constant intensities of transitions between neighboring states that have an ergodic property. Using the exponential distributions properties, we obtain formulas for the mean time of transition from the state $i$ to the state $j$ and transitions back, from the state $j$ to the state $i$. We found expressions for the mean time spent outside the given state $i$, the mean time spent in the group of states $(0, \ldots, i-1)$ to the left from state $i$, and the mean time spent in the group of states $(i+1, i+2, \ldots)$ to the right. We derive the formulas for some special cases of the Markov birth-death processes, namely, for the Erlang loss system, the queueing systems with finite and with infinite waiting room and the reliability model for a recoverable system.
\end{abstract}

MSC 2010: 60G10,60J28,60K25,93B40

Keywords: birth-death process, Markov models, mean transition time, mean time spent in the group of states, queueing systems, reliability model

\section{Introduction}

The birth-death stochastic process is one of the most important special cases of the continuous-time homogenous Markov process where the states represent the current size of a population. This process has many applications in queuing theory, reliability engineering, demography, biology and other areas [1-6]. However, establishing analytic and computationally practical formulas for their transition probabilities is usually difficult [2]. The state-of-the-art method for computing the transition probabilities of birth-death processes proposed in [3] allows for statistical estimation of general birth-death processes using likelihood-based inference [7].

As part of the practical experience of implementing various systems and analyzing their reliability indicators, it became necessary to not only estimate the availability factor and mean time between failures of the system but to also estimate the mean time $T_{i j}$ of transition from any state $i$ to any other state $j$, where $i<j$, 
as well as the average time $T_{j i}$ of transition back from state $j$ to state $i$. The need to calculate the mean values of $T_{i j}$ and $T_{j i}$ is also due to the use of birth-death models in epidemiology to predict the rate of the spread of epidemics.

In this paper, we derive analytical formulas for calculating the mean transition time between arbitrary states, the mean time spent outside the given state $i$, the mean time spent in the group of states $(0, \ldots, i-1)$ to the left from state $i$, and the mean time spent in the group of states $(i+1, i+2, \ldots)$ to the right. We obtain these formulas for some special cases of the Markov birth-death processes, namely, for the Erlang loss system, the queueing systems with finite and with infinite waiting room and the reliability model for a recoverable system.

\section{The birth-death process with an unlimited number of states}

Let us denote states by natural numbers $0,1,2, \ldots$ and assume that the intensities $\lambda_{k}, \mu_{i}$ are constant. Assume that for the birth-death process with an unlimited number of states starting from some $k$, the inequality $\lambda_{k} / \mu_{k+1} \leq \rho<1$ is satisfied.

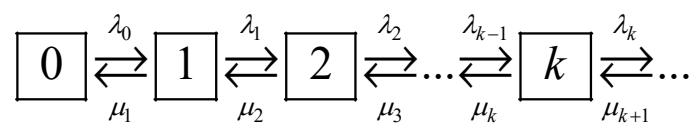

Fig. 1. Transition graph for the birth-death process with an unlimited number of states

It is well known that the distribution of the time intervals between any two successive jumps in any Markov process with continuous time and discrete space of states is exponential. More precisely, let $W_{i}$ be the instant of the $i$ th jump of the birth-death process $X(t)$ and $\tau_{i}=W_{i+1}-W_{i}$ be the sojourn time; suppose that $X\left(W_{i}\right)=k$, then the process spends exponentially distributed time $\tau_{i}$ in the state $X(t)=k$ with the mean $E\left(\tau_{i}\right)=1 /\left(\lambda_{k}+\mu_{k}\right)$. When a jump occurs, it will be a birth with the probability $\lambda_{k} /\left(\lambda_{k}+\mu_{k}\right)$ or a death with the probability $\mu_{k} /\left(\lambda_{k}+\mu_{k}\right)$.

Let us introduce notation $T_{i j}$ to denote the mean time from the instant the system comes to state $i$ to the transition to state $j$. For the birth-death process, described by the state graph shown in Figure 1, the following equations hold:

$$
T_{0 j}=\frac{1}{\lambda_{0}}+T_{1 j}, T_{k j}=\frac{1+\mu_{k} T_{k-1, j}+\lambda_{k} T_{k+1, j}}{\lambda_{k}+\mu_{k}}, 1 \leq k \leq j-2 ; T_{j-1, j}=\frac{1+\mu_{j-1} T_{j-2, j}}{\lambda_{j-1}+\mu_{j-1}} .
$$

Consistently expressing $T_{k j}$ through $T_{0 j}$ for $k \in\{1,2, \ldots, j-1\}$, we obtain the equalities 


$$
\begin{aligned}
& T_{0 j}=\sum_{k=0}^{j-1} \frac{1}{\lambda_{k}}+\sum_{k=1}^{j-1} \sum_{u=1}^{j-k} \frac{1}{\lambda_{u-1}} \prod_{s=0}^{k-1} \frac{1}{\rho_{u+s}}, j=1,2,3, \ldots ; \\
& T_{i j}=T_{0 j}-T_{0 i}=\sum_{k=i}^{j-1} \frac{1}{\lambda_{k}}+\sum_{k=1}^{j-1} \sum_{u=\max \{i-k+1,1\}}^{j-k} \frac{1}{\lambda_{u-1}} \prod_{s=0}^{k-1} \frac{1}{\rho_{u+s}}, 0 \leq i<j, j=1,2,3, \ldots,
\end{aligned}
$$

where $\rho_{s}=\lambda_{s} / \mu_{s}, s \geq 1$. Equalities (2) are valid for the processes with a finite and infinite number of states.

Suppose that $\lambda_{n+u}=\lambda_{n}, \mu_{n+u}=\mu_{n}, u \geq 1$, and $\rho_{n}=\lambda_{n} / \mu_{n}<1$. For the birth-death process described by the state graph shown in Figure 2, the following equations hold:

$$
T_{10}=\frac{1+\lambda_{1} T_{20}}{\lambda_{1}+\mu_{1}}, T_{k 0}=\frac{1+\lambda_{k} T_{k+1,0}+\mu_{k} T_{k-1,0}}{\lambda_{k}+\mu_{k}}, 2 \leq k \leq n ; T_{n+1,0}=\tilde{T}_{n}+T_{n 0},
$$

where $\tilde{T}_{n}=1 /\left(\mu_{n}-\lambda_{n}\right)$ is the mean time spent in the group of states $(n+1, n+2, \ldots)$.

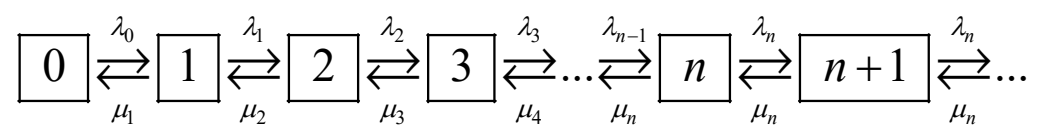

Fig. 2. Transition graph for the birth-death process with the same transition intensities starting from the state $n$

Consistently expressing $T_{k 0}$ through $T_{n+1,0}$ for $k \in\{1,2, \ldots, n\}$, we obtain the equalities

$$
\begin{aligned}
& T_{j 0}=\tilde{T}_{n} \sum_{k=1}^{j} \prod_{s=0}^{n-k} \rho_{n-s}+\sum_{k=1}^{j} \frac{1}{\mu_{k}}+\left(\sum_{k=1}^{n-j-1} \sum_{u=1}^{j}+\sum_{k=n-j}^{n-1} \sum_{u=1}^{n-k}\right) \frac{1}{\mu_{k+u}} \prod_{s=0}^{k-1} \rho_{u+s}= \\
&=\tilde{T}_{n} \sum_{k=1}^{j} \prod_{s=0}^{n-k} \rho_{n-s}+\sum_{k=1}^{j} \frac{1}{\mu_{k}}+\sum_{k=1}^{n-1} \sum_{u=1}^{\min \{j, n-k\}} \frac{1}{\mu_{k+u}} \prod_{s=0}^{k-1} \rho_{u+s}, 1 \leq j \leq n ; \\
& T_{j i}= T_{j 0}-T_{i 0}=\tilde{T}_{n} \sum_{k=i+1}^{j} \prod_{s=0}^{n-k} \rho_{n-s}+\sum_{k=i+1}^{j} \frac{1}{\mu_{k}}+\sum_{k=1}^{n-1} \sum_{u=i+1}^{\min \{j, n-k\}} \frac{1}{\mu_{k+u}} \prod_{s=0}^{k-1} \rho_{u+s}, 0 \leq i<j \leq n ; \\
& T_{n+v, 0}=T_{n 0}+v \tilde{T}_{n}, v=1,2, \ldots ; T_{n+v, i}=T_{n+v, 0}-T_{i 0}, 1 \leq i<n+v, v=1,2, \ldots .
\end{aligned}
$$

\section{The birth-death process with a finite number of states}

For the birth-death process with a finite number of states, described by the state graph shown in Figure 3, we have the equations 


$$
T_{10}=\frac{1+\lambda_{1} T_{20}}{\lambda_{1}+\mu_{1}}, T_{k 0}=\frac{1+\lambda_{k} T_{k+1,0}+\mu_{k} T_{k-1,0}}{\lambda_{k}+\mu_{k}}, 2 \leq k \leq r-1 ; T_{r 0}=\frac{1}{\mu_{r}}+T_{r-1,0} .
$$

Thus, in this case we obtain

$$
T_{j i}=\sum_{k=i+1}^{j} \frac{1}{\mu_{k}}+\sum_{k=1}^{r-1} \sum_{u=i+1}^{\min \{j, r-k\}} \frac{1}{\mu_{k+u}} \prod_{s=0}^{k-1} \rho_{u+s}, 0 \leq i<j \leq r .
$$

For the birth-death process with a finite number of states, the following equations hold:

$$
\lambda_{k} p_{k}=\mu_{k+1} p_{k+1}, \quad p_{k+1}=p_{0} \prod_{i=0}^{k} \frac{\lambda_{i}}{\mu_{i+1}}, 0 \leq k \leq r-1 ; \sum_{k=0}^{r} p_{k}=1 .
$$

Here $p_{k}$ is the steady-state probability of the system being in the state $k$. Thus, we have

$$
\begin{gathered}
p_{0}=\left(1+\sum_{k=0}^{r-1} P(0, k)\right)^{-1}, p_{k}=p_{0} P(0, k-1), 1 \leq k \leq r ; P(u, v)=\prod_{i=u}^{v} \frac{\lambda_{i}}{\mu_{i+1}} . \\
0 \underset{\mu_{1}}{\stackrel{\lambda_{0}}{\rightleftarrows}} 1 \underset{\mu_{2}}{\stackrel{\lambda_{1}}{\rightleftarrows}} 2 \underset{\mu_{3}}{\stackrel{\lambda_{2}}{\rightleftarrows}} 3 \underset{\mu_{4}}{\stackrel{\lambda_{3}}{\rightleftarrows}} \ldots \underset{\mu_{r}}{\stackrel{\lambda_{r-1}}{\rightleftarrows}} r
\end{gathered}
$$

Fig. 3. Transition graph for the birth-death process with a finite number of states

Since the steady-state probability $p_{k}$ is the relative average time, the stationary process stays in the state $k$, then

$$
p_{k}=\frac{T_{k}}{T_{k}+T_{\text {out }(k)}}, 0 \leq k \leq r, T_{0}=\frac{1}{\lambda_{0}}, T_{r}=\frac{1}{\mu_{r}} ; T_{k}=\frac{1}{\lambda_{k}+\mu_{k}}, 1 \leq k \leq r-1,
$$

where $T_{k}$ and $T_{\text {out }(k)}$ is the mean time the process stays in the state $k$ and outside the state $k$, respectively. From (8) and (9) we obtain

$$
\begin{gathered}
T_{\text {out }(0)}=\frac{1}{\lambda_{0}} \sum_{s=0}^{r-1} P(0, s), T_{\text {out }(r)}=\frac{1+\sum_{s=0}^{r-2} P(0, s)}{\mu_{r} P(0, r-1)} ; \\
T_{\text {out }(k)}=\frac{1+\sum_{\substack{s=0 \\
s \neq k-1}}^{r-1} P(0, s)}{\left(\lambda_{k}+\mu_{k}\right) P(0, k-1)}, 1 \leq k \leq r-1 .
\end{gathered}
$$


Let us denote by $T_{\text {out }(k-)}$ and $T_{\text {out }(k+)}$ the mean time that the process stays in the group of states $(0,1, \ldots, k-1)$ to the left from state $k$ and in the group of states $(k+1, k+2, \ldots, r)$ to the right from state $k$, respectively. It is obvious that

$$
T_{\text {out }(k)}=\frac{\mu_{k} T_{\text {out }(k-)}+\lambda_{k} T_{\text {out }(k+)}}{\lambda_{k}+\mu_{k}}, 1 \leq k \leq r-1 .
$$

Let us derive a formula for $T_{\text {out }(k-)}$ using the auxiliary state graph shown in Figure 4.

$$
0 \stackrel{\lambda_{\mu_{1}}}{\stackrel{\lambda_{0}}{\rightleftarrows}} 1 \underset{\mu_{2}}{\stackrel{\lambda_{1}}{\rightleftarrows}} 2 \underset{\mu_{3}}{\stackrel{\lambda_{2}}{\rightleftarrows}} \ldots \underset{\mu_{k-1}}{\stackrel{\lambda_{k-2}}{\rightleftarrows}} k-1 \underset{\mu_{k}}{\stackrel{\lambda_{k-1}}{\rightleftarrows}} k
$$

Fig. 4. Transition graph used to obtain a formula for $T_{\text {out }(k-)}$

Taking into account (8), we can write

$$
p_{k}=\frac{\mu_{k}^{-1}}{\mu_{k}^{-1}+T_{\text {out }(k-)}}=\frac{1}{1+\mu_{k} T_{\text {out }(k-)}}=\frac{P(0, k-1)}{1+\sum_{s=0}^{k-1} P(0, s)},
$$

and for the process, described by the state graph shown in Figure 3, we obtain the formulas

$$
T_{\text {out }(k-)}=\frac{1+\sum_{s=0}^{k-2} P(0, s)}{\mu_{k} P(0, k-1)}, 1 \leq k \leq r .
$$

Equalities (13) are valid for the processes with a finite and infinite number of states.

Let us derive a formula for $T_{\text {out }(k+)}$ using the auxiliary state graph shown in Figure 5 .

$$
k \underset{\mu_{k+1}}{\stackrel{\lambda_{k}}{\rightleftarrows}} k+1 \underset{\mu_{k+2}}{\stackrel{\lambda_{k+1}}{\rightleftarrows}} k+2 \underset{\mu_{k+3}}{\stackrel{\lambda_{k+2}}{\rightleftarrows}} \ldots \underset{\mu_{r}}{\stackrel{\lambda_{r-1}}{\rightleftarrows}} r
$$

Fig. 5. Transition graph used to obtain a formula for $T_{\text {out }(k+)}$

Considering (8), we have

$$
p_{k}=\frac{\lambda_{k}^{-1}}{\lambda_{k}^{-1}+T_{\text {out }(k+)}}=\frac{1}{1+\lambda_{k} T_{\text {out }(k+)}}=\frac{1}{1+\sum_{s=k}^{r-1} P(k, s)},
$$


and for the process, described by the state graph shown in Figure 3, we derive the formulas

$$
T_{\text {out }(k+)}=\frac{1}{\lambda_{k}} \sum_{s=k}^{r-1} P(k, s), 0 \leq k \leq r-1 .
$$

For the process with an unlimited number of states, relations (15) can be written as

$$
T_{\text {out }(k+)}=\frac{1}{\lambda_{k}} \sum_{s=k}^{\infty} P(k, s), k \geq 0 .
$$

Using equalities

$$
T_{i j}=T_{\text {out }(i+1,-)}+T_{i+1, j}, T_{j i}=T_{\text {out }(j-1,+)}+T_{j-1, i}, 0 \leq i<j,
$$

that follow from the properties of the Markov process, we obtain the following formulas

$$
\begin{aligned}
& T_{\text {out }(k-)}=T_{k-1, j}-T_{k j}, 1 \leq k<j ; T_{\text {out }(k+)}=T_{k+1, i}-T_{k i}, 0 \leq i<k ; \\
& T_{i j}=\sum_{k=i+1}^{j} T_{\text {out }(k-)}, T_{j i}=\sum_{k=i}^{j-1} T_{\text {out }(k+)}, 0 \leq i<j .
\end{aligned}
$$

Equalities (18) are valid for the processes with a finite and infinite number of states.

Using formulas (18), we obtain simpler expressions for $T_{i j}$ and $T_{j i}$ than we have in (2) and (6):

$$
\begin{aligned}
& T_{i j}=\sum_{k=i+1}^{j} T_{\text {out }(k-)}=\sum_{k=i+1}^{j} \frac{1+\sum_{s=0}^{k-2} P(0, s)}{\mu_{k} P(0, k-1)}, 0 \leq i<j ; \\
& T_{j i}=\sum_{k=i}^{j-1} T_{\text {out }(k+)}=\sum_{k=i}^{j-1} \frac{1}{\lambda_{k}} \sum_{s=k}^{r-1} P(k, s), 0 \leq i<j \leq r .
\end{aligned}
$$

\section{The Erlang loss queueing system}

The Erlang loss model is usually used to obtain the number a telephone network links. In the paper [8] this model is applied for describing in-patient flow through a hospital ward.

For the Erlang loss queueing system (see Fig. 6), changing the number of busy servers is a birth-death process with a finite number of states and the following values of the transition intensities

$$
\lambda_{0}=\lambda, \lambda_{k}=\lambda, \mu_{k}=k \mu, 1 \leq k \leq n
$$




$$
0 \underset{\mu}{\stackrel{\lambda}{\rightleftarrows}} 1 \underset{2 \mu}{\stackrel{\lambda}{\rightleftarrows}} 2 \underset{3 \mu}{\stackrel{\lambda}{\rightleftarrows}} \stackrel{2}{\underset{4 \mu}{\rightleftarrows}} \ldots \underset{n \mu}{\stackrel{\lambda}{\rightleftarrows}} n
$$

Fig. 6. Transition graph for the loss queueing system

In this case, formulas (10), (13), (15) and (19) can be written as

$$
\begin{aligned}
& T_{i j}=\sum_{k=i+1}^{j} T_{\text {out }(k-)}=\frac{1}{\mu} \sum_{k=i+1}^{j} \frac{(k-1) !}{\rho^{k}} \sum_{s=0}^{k-1} \frac{\rho^{s}}{s !}, 0 \leq i<j \leq n ; \\
& T_{j i}=\sum_{k=i}^{j-1} T_{\text {out }(k+)}=\frac{1}{\lambda} \sum_{k=i}^{j-1} k ! \sum_{s=k+1}^{n} \frac{\rho^{s-k}}{s !}, 0 \leq i<j \leq n ; \\
& T_{\text {out }(k)}=\frac{k !}{(\lambda+k \mu) \rho^{k}} \sum_{\substack{s=0 \\
s \neq k}}^{n} \frac{\rho^{s}}{s !}, 0 \leq k<n ; T_{\text {out }(n)}=\frac{(n-1) !}{\mu \rho^{n}} \sum_{s=0}^{n-1} \frac{\rho^{s}}{s !} ; \\
& T_{\text {out }(k-)}=\frac{(k-1) !}{\mu \rho^{k}} \sum_{s=0}^{k-1} \frac{\rho^{s}}{s !}, 1 \leq k \leq n ; T_{\text {out }(k+)}=\frac{k !}{\lambda} \sum_{s=k+1}^{n} \frac{\rho^{s-k}}{s !}, 0 \leq k \leq n-1,
\end{aligned}
$$

where $\rho=\lambda / \mu$.

For practical applications, it is important to know the average system availability time $T_{\text {out }(n)}$, when at least one server is free, the mean busy period $T_{\text {out }(0)}$, and the values of $T_{0 n}$ and $T_{n 0}$.

For the case of unlimited number of servers $(n \rightarrow \infty)$, equalities (21) can be written as

$$
\begin{aligned}
& T_{i j}=\sum_{k=i+1}^{j} T_{\text {out }(k-)}=\frac{1}{\mu} \sum_{k=i+1}^{j} \frac{(k-1) !}{\rho^{k}} \sum_{s=0}^{k-1} \frac{\rho^{s}}{s !}, 0 \leq i<j ; \\
& T_{j i}=\sum_{k=i}^{j-1} T_{\text {out }(k+)}=\frac{1}{\lambda} \sum_{k=i}^{j-1} \frac{k !}{\rho^{k}}\left(e^{\rho}-\sum_{s=0}^{k} \frac{\rho^{s}}{s !}\right), 0 \leq i<j ; \\
& T_{\text {out }(k)}=\frac{k !}{(\lambda+k \mu) \rho^{k}}\left(e^{\rho}-\frac{\rho^{k}}{k !}\right), k \geq 0 ; \\
& T_{\text {out }(k-)}=\frac{(k-1) !}{\mu \rho^{k}} \sum_{s=0}^{k-1} \frac{\rho^{s}}{s !}, k \geq 1 ; T_{\text {out }(k+)}=\frac{k !}{\lambda \rho^{k}}\left(e^{\rho}-\sum_{s=0}^{k} \frac{\rho^{s}}{s !}\right), k \geq 0 .
\end{aligned}
$$

\section{Queueing systems with finite and infinite waiting room}

For the queueing system with a finite waiting room capacity (see Fig. 7), the number of customers in the system is a birth-death process with a finite number of states and the following values of the transition intensities

$$
\lambda_{k}=\lambda, 0 \leq k \leq r ; \mu_{k}=k \mu, 1 \leq k \leq n ; \mu_{k}=n \mu, n+1 \leq k \leq r .
$$




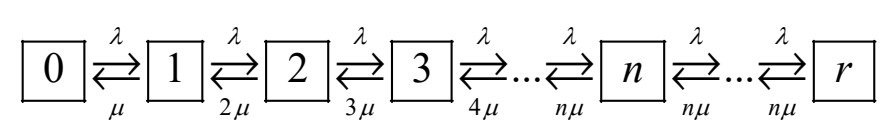

Fig. 7. Transition graph for the queueing system with finite waiting room capacity

Let us denote $\beta=\lambda /(n \mu)$, then, using (2), (6), (10), (13), (15) and (19), we obtain

$$
\begin{aligned}
& T_{\text {out }(k)}=\frac{k !}{n !(\lambda+k \mu)}\left(n ! \sum_{\substack{s=0 \\
s \neq k}}^{n} \frac{\rho^{s-k}}{s !}+\rho^{n-k} \sum_{s=1}^{r-n} \beta^{s}\right), 0 \leq k \leq n ; \\
& T_{\text {out }(k)}=\frac{1}{(\lambda+n \mu) \rho^{n} \beta^{k-n}}\left(n ! \sum_{s=0}^{n} \frac{\rho^{s}}{s !}+\rho^{n} \sum_{s=1}^{k-n-1} \beta^{s}+\rho^{n} \beta^{k-n} \sum_{s=1}^{r-k} \beta^{s}\right), n+1 \leq k<r ; \\
& T_{\text {out }(k-)}=\frac{(k-1) !}{\mu \rho^{k}} \sum_{s=0}^{k-1} \frac{\rho^{s}}{s !}, 1 \leq k \leq n \text {; } \\
& T_{\text {out }(k-)}=\frac{1}{\mu} \frac{n ! \sum_{s=0}^{n} \frac{\rho^{s}}{s !}+\rho^{n} \sum_{s=1}^{k-n-1} \rho^{s}}{n \rho^{n} \beta^{k-n}}, n+1 \leq k \leq r \\
& T_{\text {out }(k+)}=\frac{k !}{n ! \lambda}\left(n ! \sum_{s=k+1}^{n} \frac{\rho^{s-k}}{s !}+\rho^{n-k} \sum_{s=1}^{r-n} \beta^{s}\right), 0 \leq k \leq n-1 \\
& T_{\text {out }(k+)}=\frac{1}{\lambda} \sum_{s=1}^{r-k} \beta^{s}, n \leq k \leq r-1 \\
& T_{i j}=\sum_{k=i+1}^{j} T_{\text {out }(k-)}=\frac{1}{\mu} \sum_{k=i+1}^{j} \frac{(k-1) !}{\rho^{k}} \sum_{s=0}^{k-1} \frac{\rho^{s}}{s !}, 0 \leq i<j \leq n ; \\
& T_{i j}=\frac{1}{\mu} \sum_{k=i+1}^{n} \frac{(k-1) !}{\rho^{k}} \sum_{s=0}^{k-1} \frac{\rho^{s}}{s !}+\frac{1}{\mu} \sum_{k=n+1}^{j} \frac{n ! \sum_{s=0}^{n} \frac{\rho^{s}}{s !}+\rho^{n} \sum_{s=1}^{k-n-1} \rho^{s}}{n \rho^{n} \beta^{k-n}}, 0 \leq i \leq n<j ; \\
& T_{i j}=\frac{1}{\mu} \sum_{k=i+1}^{j} \frac{n ! \sum_{s=0}^{n} \frac{\rho^{s}}{s !}+\rho^{n} \sum_{s=1}^{k-n-1} \rho^{s}}{n \rho^{n} \beta^{k-n}}, n<i<j \leq r \\
& T_{j i}=\sum_{k=i}^{j-1} T_{\text {out }(k+)}=\frac{1}{n ! \lambda} \sum_{k=i}^{j-1} k !\left(n ! \sum_{s=k+1}^{n} \frac{\rho^{s-k}}{s !}+\rho^{n-k} \sum_{s=1}^{r-n} \beta^{s}\right), 0 \leq i<j \leq n ; \\
& T_{j i}=\frac{1}{n ! \lambda} \sum_{k=i}^{n-1} k !\left(n ! \sum_{s=k+1}^{n} \frac{\rho^{s-k}}{s !}+\rho^{n-k} \sum_{s=1}^{r-n} \beta^{s}\right)+\frac{1}{\lambda} \sum_{k=n}^{j-1} \sum_{s=1}^{r-k} \beta^{s}, 0 \leq i<n<j \leq r ; \\
& T_{j i}=\sum_{k=i}^{j-1} T_{o u t(k+)}=\frac{1}{\lambda} \sum_{k=i}^{j-1} \sum_{s=1}^{r-k} \beta^{s}, n \leq i<j \leq r .
\end{aligned}
$$


Those of equalities (24)-(25) which do not contain $r$ remain valid for the queueing system with an infinite waiting room capacity for which we assume that $\beta<1$. In other cases, we need to calculate the limit as $r \rightarrow \infty$, and we obtain

$$
\begin{aligned}
& T_{\text {out }(k)}=\frac{k !}{n !(\lambda+k \mu)}\left(n ! \sum_{\substack{s=0 \\
s \neq k}}^{n} \frac{\rho^{s-k}}{s !}+\rho^{n-k} \frac{\beta}{1-\beta}\right), 0 \leq k \leq n \\
& T_{\text {out }(k)}=\frac{1}{(\lambda+n \mu) \rho^{n} \beta^{k-n}}\left(n ! \sum_{s=0}^{n} \frac{\rho^{s}}{s !}+\rho^{n} \sum_{s=1}^{k-n-1} \beta^{s}+\rho^{n} \frac{\beta^{k+1-n}}{1-\beta}\right), k \geq n+1 ; \\
& T_{\text {out }(k+)}=\frac{k !}{n ! \lambda}\left(n ! \sum_{s=k+1}^{n} \frac{\rho^{s-k}}{s !}+\frac{\rho^{n-k} \beta}{1-\beta}\right), 0 \leq k \leq n-1 ; \\
& T_{\text {out }(k+)}=\frac{\beta}{\lambda(1-\beta)}, k \geq n ; \quad n=\frac{1}{n ! \lambda} \sum_{k=i}^{j-1} k !\left(n ! \sum_{s=k+1}^{n} \frac{\rho^{s-k}}{s !}+\frac{\rho^{n-k} \beta}{1-\beta}\right), 0 \leq i<j \leq n ; \\
& T_{j i}=\sum_{k=i}^{j-1} T_{\text {out }(k+)}=\frac{1}{n ! \lambda} \sum_{k=i}^{n-1} k !\left(n ! \sum_{s=k+1}^{n} \frac{\rho^{s-k}}{s !}+\frac{\rho^{n-k} \beta}{1-\beta}\right)+\frac{(j-n) \beta}{\lambda(1-\beta)}, 0 \leq i<n<j ; \\
& T_{j i}=\frac{(j-i) \beta}{\lambda(1-\beta)}, n \leq i<j .
\end{aligned}
$$

For practical applications, it is important to know the average system availability time $T_{\text {out }(r)}$, the mean busy period $T_{\text {out }(0)}$, and the values of $T_{0 r}$ and $T_{r 0}$.

\section{Series recoverable system with redundancy and repair facilities}

Let us consider a Markov model for studying the reliability of series systems with redundancy and repair facilities.

Consider a system that consists of $r=m+c$ identical units, namely, $m$ main operating units and $c$ unloaded redundant units. The main units are connected in a series. The system stops functioning in normal mode at the moment when the number of failed units reaches $c+1$. Assume that those $m-1$ units that were serviceable at the moment of system failure, in idle time during recovery, continue to operate and may fail. Let us suppose that the number $n$ of repair facilities is restricted $(n \leq c)$, so failed units can form a queue for recovering. 
Let us denote states by natural numbers $0,1,2, \ldots$, where the number of a state corresponds to the number of failed units. Then the state graphs have the form shown in Figure 8.

$$
0 \underset{\mu}{\stackrel{m \lambda}{\rightleftarrows}} 1 \underset{2 \mu}{\stackrel{m \lambda}{\rightleftarrows}} 2 \underset{3 \mu}{\stackrel{m \lambda}{\rightleftarrows}} \ldots \underset{n \mu}{\stackrel{m \lambda}{\rightleftarrows}} n \underset{n \mu}{\stackrel{m \lambda}{\rightleftarrows}} \ldots \underset{n \mu}{\stackrel{m \lambda}{\rightleftarrows}} c \underset{n \mu}{\stackrel{m \lambda}{\rightleftarrows}} c+1 \underset{n \mu}{\stackrel{(m-1) \lambda}{\rightleftarrows}} \ldots \underset{n \mu}{\stackrel{\lambda}{\rightleftarrows}} r
$$

Fig. 8. Transition graph for the series system with redundancy and repair facilities

The number of failed units is a birth-death process with a finite number of states and the following values of the transition intensities

$$
\begin{aligned}
& \lambda_{k}=m \lambda, 0 \leq k \leq c ; \lambda_{k}=(r-k) \lambda, c+1 \leq k \leq r-1 ; \\
& \mu_{k}=k \mu, 1 \leq k \leq n ; \mu_{k}=n \mu, n+1 \leq k \leq r .
\end{aligned}
$$

Here $\lambda$ and $\mu$ are the parameters of exponential distributions of the time to failure and the recovery time respectively. For this system, the time to failure $X_{S}$ and the time between failures $\tilde{X}_{S}$ do not coincide, because the time interval $X_{S}$ begins at the moment of transition from state 1 to state 0 , and the time $\tilde{X}_{S}$ begins at the moment of transition from state $c+1$ to state $c$. Both states end simultaneously at the moment of transition from the state $c$ to the state $c+1$. The downtime $X_{S I}$ is the time spent in a group of states $(c+1, \ldots, r)$.

With the help of (13) and (15), we obtain

$$
\begin{aligned}
T_{\text {out }(k-)}= & \frac{(k-1) !}{\mu(m \rho)^{k}} \sum_{s=0}^{k-1} \frac{(m \rho)^{s}}{s !}, 1 \leq k \leq n+1 ; \\
T_{\text {out }(k-)}= & \frac{(n-1) ! n^{k-n}}{\mu(m \rho)^{k}}\left(\sum_{s=0}^{n} \frac{(m \rho)^{s}}{s !}+\sum_{s=n+1}^{k-1} \frac{(m \rho)^{s}}{n ! n^{s-n}}\right), n+2 \leq k \leq c+1 ; \\
T_{\text {out }(k-)}= & \frac{(n-1) !(r-k) ! n^{k-n}}{m ! m^{c} \mu \rho^{k}} \times \\
& \times\left(\sum_{s=1}^{n} \frac{(m \rho)^{s}}{s !}+\sum_{s=n+1}^{c+1} \frac{(m \rho)^{s}}{n ! n^{s-n}}+\sum_{s=c+2}^{k-1} \frac{m ! m^{c} \rho^{s}}{n !(r-s) ! n^{s-n}}\right), c+2 \leq k \leq r ; \\
T_{\text {out }(k+)}= & \frac{k !}{\lambda m^{k+1} \rho^{k}}\left(\sum_{s=k+1}^{n} \frac{(m \rho)^{s}}{s !}+\sum_{s=n+1}^{c+1} \frac{(m \rho)^{s}}{n ! n^{s-n}}+\sum_{s=c+2}^{r} \frac{m ! m^{c} \rho^{s}}{n !(r-s) ! n^{s-n}}\right), 0 \leq k \leq n-1 ; \\
T_{\text {out }(k+)}= & \frac{n^{k}}{\lambda m^{k+1} \rho^{k}}\left(\sum_{s=k+1}^{c+1} \frac{(m \rho)^{s}}{n^{s}}+\sum_{s=c+2}^{r} \frac{m ! m^{c} \rho^{s}}{(r-s) ! n^{s}}\right), n \leq k \leq c ; \\
T_{\text {out }(k+)}= & \frac{(r-k-1) ! n^{k}}{\lambda \rho^{k}} \sum_{s=k+1}^{r} \frac{\rho^{s}}{(r-s) ! n^{s}}, c+1 \leq k \leq r-1 .
\end{aligned}
$$


Using expressions (29) and equalities (19), we can derive formulas for $T_{i j}$ and $T_{j i}, 0 \leq i<j \leq r$. We present only formulas for the mean values $E\left(X_{S}\right)=T_{0, c+1}$, $E\left(\tilde{X}_{S}\right)=T_{c, c+1}$ and $E\left(X_{S I}\right)=T_{\text {out }(c+)}$ :

$$
\begin{aligned}
E\left(X_{S}\right) & =T_{0, c+1}=\sum_{k=1}^{c+1} T_{\text {out }(k-)}=\frac{1}{\mu}\left(\sum_{k=1}^{n+1} \frac{(k-1) !}{(m \rho)^{k}} \sum_{s=0}^{k-1} \frac{(m \rho)^{s}}{s !}+\right. \\
& \left.+\sum_{k=n+2}^{c+1} \frac{(n-1) ! n^{k-n}}{(m \rho)^{k}}\left(\sum_{s=0}^{n} \frac{(m \rho)^{s}}{s !}+\sum_{s=n+1}^{k-1} \frac{(m \rho)^{s}}{n ! n^{s-n}}\right)\right) ; \\
E\left(\tilde{X}_{S}\right)= & T_{c, c+1}=T_{\text {out }(c+1-)}=\frac{(n-1) ! n^{c+1-n}}{\mu(m \rho)^{c+1}}\left(\sum_{s=0}^{n} \frac{(m \rho)^{s}}{s !}+\sum_{s=n+1}^{c} \frac{(m \rho)^{s}}{n ! n^{s-n}}\right) ; \\
E\left(X_{S I}\right)= & T_{\text {out }(c+)}=\frac{1}{n \mu}+\frac{n^{c}(m-1) !}{\lambda \rho^{c}} \sum_{s=c+2}^{r} \frac{\rho^{s}}{(r-s) ! n^{s}} .
\end{aligned}
$$

\section{Conclusions}

Using the exponential distributions properties and peculiarities of the Markov process we have established a connection between the transition times between the states of the birth-death process and the times spent in the group of states. This indicates an easy method to get formulas for average "travel" times between the states of this process.

The obtained formulas can be used to test simulation models $[9,10]$ built to calculate the characteristics of various stochastic systems. The constructed simulation models can be used for systems with non-exponential distributions.

\section{References}

[1] Ushakov, I. (2012). Probabilistic Reliability Models. Hoboken: John Wiley \& Sons.

[2] Novozhilov, A.S., Karev, G.P., \& Koonin, E.V. (2006). Biological applications of the theory of birth-and-death processes. Briefings in Bioinformatics, 7(1), 70-85.

[3] Crawford, F.W., \& Suchard, M.A. (2012). Transition probabilities for general birth-death processes with applications in ecology, genetics, and evolution. Journal of Mathematical Biology, 65(3), 553-580.

[4] Doss, C.R., Suchard, M.A., Holmes, I., Kato-Maeda, M., \& Minin, V.N. (2013). Fitting birth-death processes to panel data with applications to bacterial DNA fingerprinting. The Annals of Applied Statistics, 7(4), 2315-2335.

[5] Rabier, C.-E., Ta, T., \& Ané C. (2014). Detecting and locating whole genome duplications on a phylogeny: a probabilistic approach. Molecular Biology and Evolution, 31(3), 750-762. 
[6] Crawford, F.W., Weiss, R.E., \& Suchard, M.A. (2015). Sex, lies, and self-reported counts: Bayesian mixture models for longitudinal heaped count data via birth-death processes. Annals of Applied Statistics, 9, 572-596.

[7] Crawford, F.W., Minin, V.N., \& Suchard, M.A. (2014). Estimation for general birth-death processes. Journal of the American Statistical Association, 109(506), 730-747.

[8] De Bruin, A.M., Bekker, R., van Zanten, L., \& Koole, G.M. (2010). Dimensioning hospital wards using the Erlang loss model. Annals of Operations Research, 178(1), 23-43.

[9] Zhernovyi, Yu. (2015). Creating Models of Queueing Systems Using GPSS World. Saarbrücken: LAP Lambert Academic Publishing.

[10] Zhernovyi, Yu.V. (2020). Simulation Models of Reliability. Zhytomyr: PC "Zhytomyr-Polygraph" (in Ukrainian). 